\title{
Structure Design and Experimental Study of Pin-Cycloidal Planetary Reducer for the Pitch of High-Power Wind Turbine
}

\author{
Lu Qi*, He Weidong \\ Mechanical Engineering,Dalian Jiaotong University, Dalian, Liaoning, China \\ *Corresponding Author.
}

\begin{abstract}
In recent years, with the development of manufacturing technology and production capacity, wind power industry has been developed rapidly.A new type of pin-cycloidal planetary reducer was proposed in this studyfor the actuator of the pitch of high-power wind turbines. Based on the transformation mechanism method, the transmission efficiency of the reducer was calculated by analyzing the pin-cycloidal meshing efficiency, bearing efficiency, and output mechanism efficiency. Prototype testing were combined to systematically and deeply study the design theory of the reducer for the pitch of high-power wind turbines. Finally, a new design was proposed for a reliable two-stage pin-cycloidal planetary reducer with a strong bearing capacity and multi-tooth meshing on the hard gear surface that can be applied to the pitch system of high-power wind turbines.
\end{abstract}

Keywords:wind power industry, pin-cycloidal, pitch, transmission efficiency, prototype testing

\section{Introduction}

As one of the key components of the high-power wind power generation system, the pitch adjusts the pitch angle of the blades mounted on the wheel hub of the wind turbine at the appropriate time. The pitch angle of the blades influences significantly the wind energy absorbed by the wind rotor close to the rated power, and it can also effectively reduce the strong impact on the wind turbine when the wind speed changes drastically [1]. According to the different pitch technologies of the blade, a pitch system can be classified as a hydraulic pitch or an electric pitch [2], the latter of which is composed of a power source motor, a control module, a battery, and an actuator reducer.Figure 1 shows theadjustment of pitch angle of the blade.

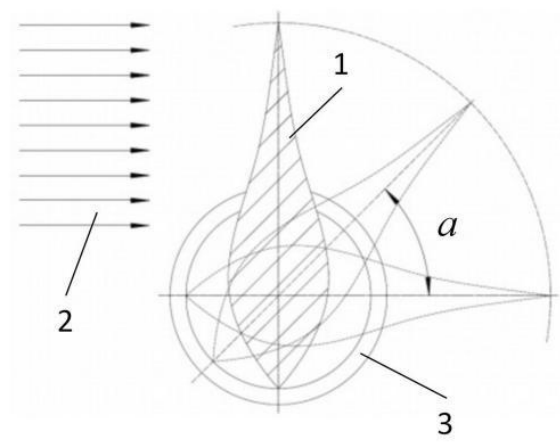

1. Blade; 2. Airflow; 3. Wheel hub

Fig 1: The adjustment of pitch angle of the blade

Currently, the actuator reducer of the pitch of high-power wind turbines universally adopts the multi-stage involute planetary gear transmission mechanism [3]. Compared with the involute gear transmission, the pin-cycloidal transmission has significant advantages, e.g., simultaneous meshing of multiple teeth, large single-stage transmission ratio, high transmission efficiency, small size, low weight, high reliability, stable transmission, and low noise [4]. 


\section{Structural Design of the Two-Stage Pin-Cycloidal Planetary Reducer}

\subsection{Transmission principle}

The figure 2 shows the transmission principle of the two-stage pin-cycloidal planetary reducer. Input shaft 2 is fixed with an eccentric bushing. When input shaft 2 rotates counterclockwise, cycloidal gears 1 and 3 with a phase difference of $180^{\circ}$ in stage I are rotated by the turning-arm bearing on the eccentric bushing. The cycloidal gears mesh the pin gear fixed on pin wheel housing 4 in stage I, and the axis of cycloidal gears 1 and 3 thereof revolves around the axis of the pin wheel while rotating in the opposite direction (clockwise). Cycloidal gears1 and 3 in stage I rotate to drive the pins that are evenly inserted into the pin holes in intermediate shaft 6 to rotate, thereby driving intermediate shaft 6 to rotate clockwise. Intermediate shaft 6 serves as the output shaft of the deceleration system in stage I as well as the input shaft of the deceleration system in stage II. The transmission mode in stage II is the same as that in stage I, and the output transmission direction in stage II is counterclockwise.

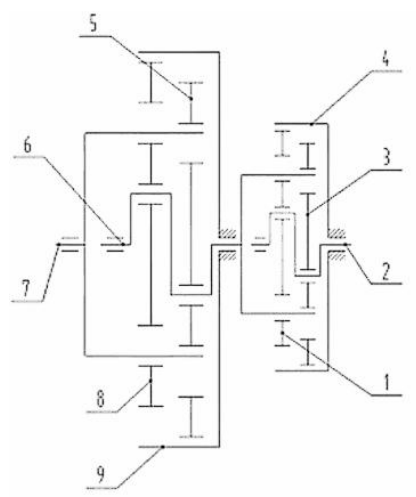

1. Left cycloidalgear in stage I; 2. Input shaft; 3. Right cycloidalgear in stage I; 4. Pin wheel housing in stage I; 5. Right cycloidalgear in stage II; 6. Intermediate shaft; 7. Output shaft; 8. Left cycloidalgear in stage II; 9. Pin wheel housing in stage II

1. Blade; 2. Airflow; 3. Wheel hub

Fig 2: Transmission principle of the two-stage pin-cycloidal planetary reducer

\subsection{Force analysis}

An accurate force analysis was performed on the cycloidal gear, output mechanism, turning-arm bearing, input shaft, intermediate shaft, and output shaft, which not only laid the foundation for calculating the reliability of the whole machine but also determined the service life of each bearing in the reducer, including the turning-arm bearing and other support bearings. Figure 3 shows the force state analysis of the cycloidalgear in stage I, and Figure 4 shows the force state analysis of the output mechanism in stage II.
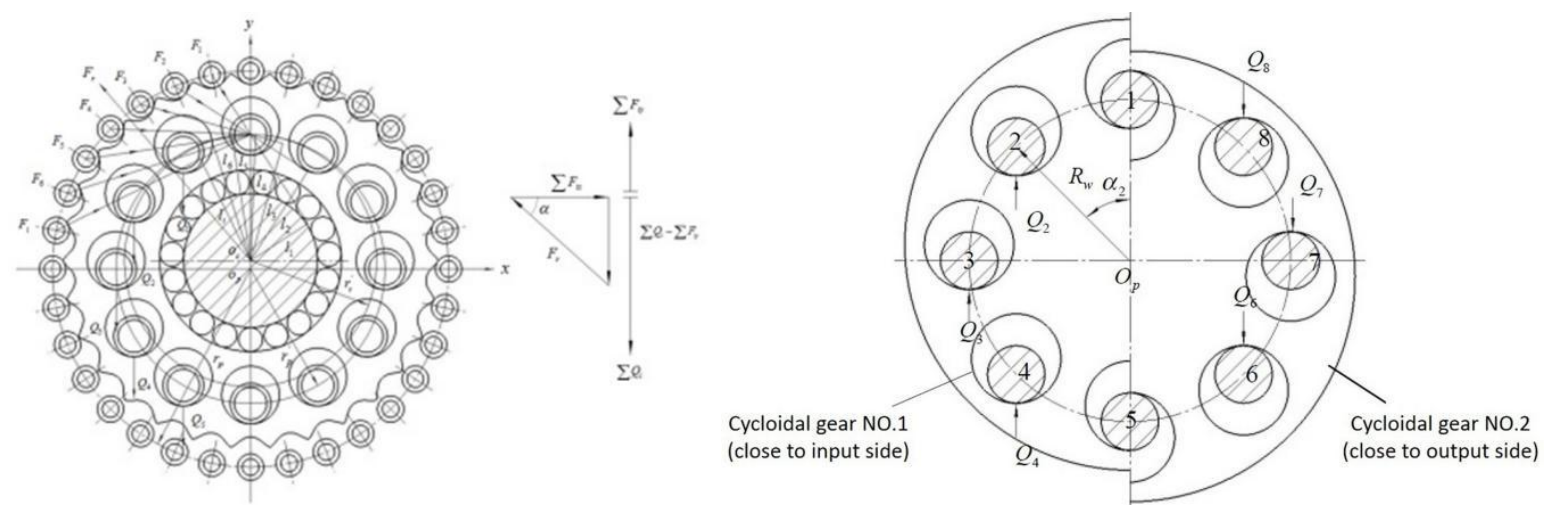

Fig 3: Force state analysis of cycloidalgear in stage IFig 4: Force state analysis of output mechanism in stage II

ISSN: 0010-8189

(C) CONVERTER 2020

Www.converter-magazine.info 


\subsection{Structure design}

According to the optimized transmission parameters of the pin-cycloidal planetary reducer, and to meet the structural interchangeability requirements (that is, the external connection size must meet the installation conditions of the current product), the structure of the pin-cycloidal planetary reducer for the pitch was determined (Figure 5).

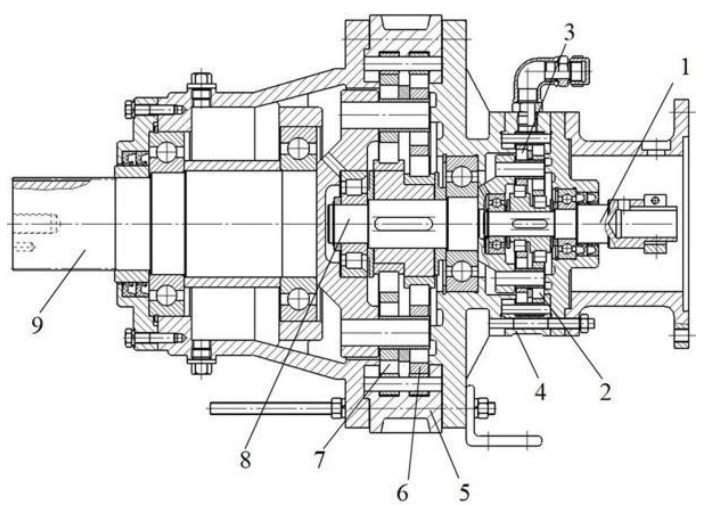

1.Input shaft; 2.Cycloidal gear NO.1 of stage I; 3.Cycloidal gear NO.2 of stage I; 4.Pin house of stage I; 5.Pin house of stage II; 6.Cycloidal gear NO.1 of stage II; 7.Cycloidal gear NO.2 of stage II; 8.Middle shaft; 9.Output shaft.

Fig 5: Structure of the pin-cycloidal planetary reducer for the pitch

The reducer adopts a two-stage pin-cycloidal meshing planetary transmission. The two cycloidal gears in the reducer in each stage mechanism are in a tooth profile of "two-teeth difference" with a phase difference of $180^{\circ}$, and they were each modified with a "one-tooth difference" cycloidal tooth profile to remove the sharp points[5]. The reduction ratio in stage $\mathrm{I}$ is 11 , the reduction ratio in stage II is 15 , and that of the whole machine is 165 . An integral double eccentric bearing was used as the turning-arm bearing in stage I, whereas because of the limited radial dimension in stage II, a combination of an eccentric bushing and a cylindrical roller bearing was used as the turning-arm bearing in stage II. The transmission torque load in stage II is relatively large, so in order to reduce the bending and deformation of the pin gear, the pin gear was installed in this deceleration system with three supporting points. The input end and the output end of the reducer were sealed by a double-frame oil seal, and the input motor was directly mounted on the motor bracket of the reducer. Compared with the three-stage involute gear planetary reducer, the product currently used in the pitch actuator, the whole machine was reduced by $63.5 \mathrm{~mm}$ in axial dimension and decreased by $22 \mathrm{~kg}$ in mass.

\section{Transmission Efficiency}

By analyzing the pin-cycloidal meshing efficiency, bearing efficiency, and efficiency of the output mechanism, the transmission efficiency of the pin-cycloidal planetary reducer for pitch control was calculated[6].

\subsection{Efficiency of meshing}

The transmission mechanism is shown in Figure 6 , wherez $z_{p}$ is the number of teeth of the pin gear, which rotates clockwise at an angular speed of $\omega_{b} . z_{a}$ is the number of the two-teeth difference cycloidal gear teeth, and $\omega_{a}$ represents its angular speed. 

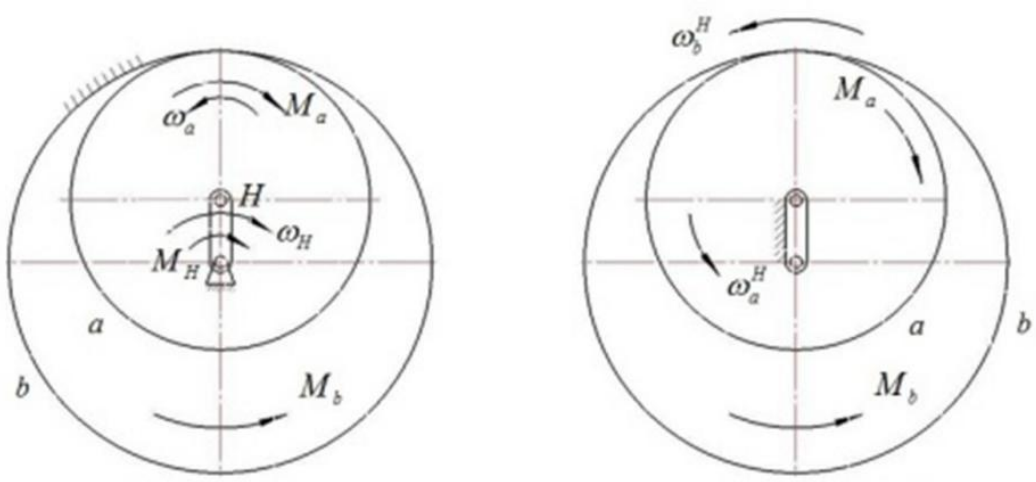

Fig 6:Planetary mechanism (a) and transmission mechanism (b)

In the planetary mechanism, $\mathrm{H}$ denotes the driving gear and $\alpha$ denotes the driven gear, whose meshing efficiency $\eta_{H a}^{b}$ can be expressed as follows:

$$
\eta_{H a}^{b}=-\frac{M_{a} \omega_{a}^{b}}{M_{H} \omega_{H}^{b}}=\frac{\eta^{H}\left(1-i_{a b}^{H}\right)}{\eta^{H}-i_{a b}^{H}}
$$

The meshing efficiency of the stage-I transformation mechanism, $\eta_{1}^{\mathrm{H}}$ is:

$$
\eta_{\mathrm{I}}^{H}=1-\frac{8 f r_{z \mathrm{I}}^{\prime}}{k_{1 \mathrm{I}} Z_{a \mathrm{I}} Z_{p \mathrm{I}} r_{z \mathrm{I}}}\left(1-\frac{r_{z \mathrm{I}}}{R_{z \mathrm{I}}}\right) \sum_{i=1}^{6} \sin \varphi_{i \mathrm{I}}(2)
$$

The meshing efficiency of the stage-II transformation mechanism, $\eta_{\Pi}^{\mathrm{H}}$ is:

$$
\eta_{\mathrm{II}}^{H}=1-\frac{8 f r_{z \mathrm{II}}^{\prime}}{k_{1 \mathrm{II}} Z_{a \mathrm{II}} Z_{p \mathrm{II}} r_{z \mathrm{II}}}\left(1-\frac{r_{z \mathrm{II}}}{R_{z \mathrm{II}}}\right) \sum_{i=2}^{8} \sin \varphi_{i \mathrm{II}}(3)
$$

According to Equation (2), $\eta_{1}^{\mathrm{H}}=0.99951$; based on Equation (3), $\eta_{\Pi}^{\mathrm{H}}=0.99969$. Through the calculation of Equation $(1), \eta_{\mathrm{Ha}}^{\mathrm{b}}$, the meshing efficiency of the two planetary mechanisms, were obtained, which were denoted by $\eta_{\mathrm{NI}}$ and $\eta_{\mathrm{N}}$, respectively. Then the meshing efficiency of the planetary mechanism can be expressed as follows:

$$
\eta_{N}=\eta_{N \mathrm{I}} \eta_{N \mathrm{II}}
$$

\subsection{Efficiency of the output mechanism}

The figure 7 shows the relative sliding speed of the pin hole of the cycloidal gear and the pin of the output disk pin at the contact point $\mathrm{E}$ in the output mechanism. 


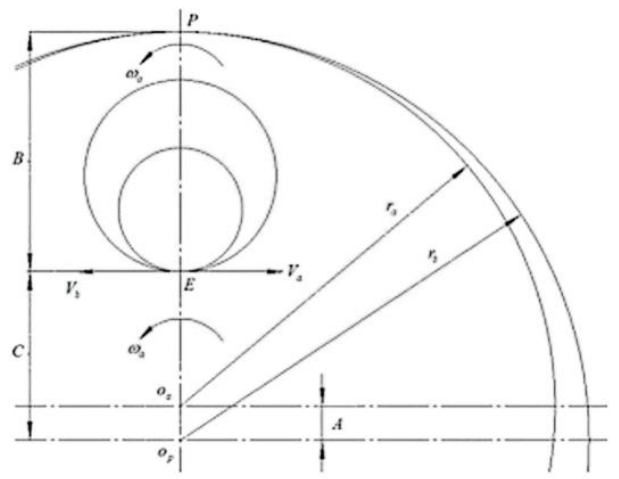

Fig 7:Force of the cycloidal gear on the pin in the output mechanism

The friction power consumed in the output mechanism (W mechanism) is:

$$
N_{W T}=\frac{4 T_{a}}{\pi R_{w}} A\left|\omega_{H}-\omega_{a}\right| f_{w} \frac{r_{p}^{\prime}}{r_{p}}(5)
$$

The efficiency of the output mechanism is:

\subsection{Efficiency of bearing}

$$
\eta_{W}=\frac{\omega_{a} T_{a}-N_{W T}}{\omega_{a} T_{a}}=1-\frac{4 f_{w} A r_{p}^{\prime}}{\pi R_{w} r_{p}} \cdot\left|\frac{\omega_{H}-\omega_{a}}{\omega_{a}}\right| \text { (6) }
$$

Since the angle between the radial force on the turning-arm bearing and the circumferential force on the turning arm is large, the radial load acting on the turning-arm bearing is large as well, so the transmission efficiency of the turning-arm bearing is $\eta_{B}$ that equals to 0.985 . The transmission efficiencies of the support bearings, including the support bearings of the input shaft (deep groove ball bearings), the support bearings of the intermediate shaft (deep groove ball bearings + cylindrical roller bearings), and the support bearings of the output shaft (deep groove ball bearings), were calculated according to the following equation:

$$
\eta_{B}^{\prime}=\eta_{B 1}^{\prime} \eta_{B 2}^{\prime} \eta_{B 3}^{\prime}(7)
$$

\subsection{Transmission Efficiency of the Whole Machine}

Based on the above calculation results, $\eta$ The transmission efficiency of the pin-cycloidal planetary reducer for the pitch, can be expressed as:

$$
\eta=\eta_{N} \eta_{B} \eta_{W} \eta_{B}^{\prime} \eta_{V}
$$

Where $\eta_{V}$ is the efficiency consumed by oil mixing, and if grease is used, then $\eta_{V}=1$ [7].

During the transmission process of the whole machine, since relative sliding and rolling occurred between the tooth profile of the cycloidal gear and the tooth profile of the pin gear, sliding friction and rolling friction were present. The rolling friction between the wheel roller and the cycloidal gear tooth is so small that it can be ignored. With only the sliding friction loss between the wheel roller and the wheel pin considered, the transmission efficiency of the pin-cycloidal planetary reducer calculated per Equation (8) is 0.9078.

\section{Experimental Research}

ISSN: 0010-8189

(C) CONVERTER 2020

Www.converter-magazine.info 
Figure 8 shows the experimental devices for the pin-cycloidal planetary reducer for the pitch of high-power wind turbines. Figure 9 shows the test bench solution.

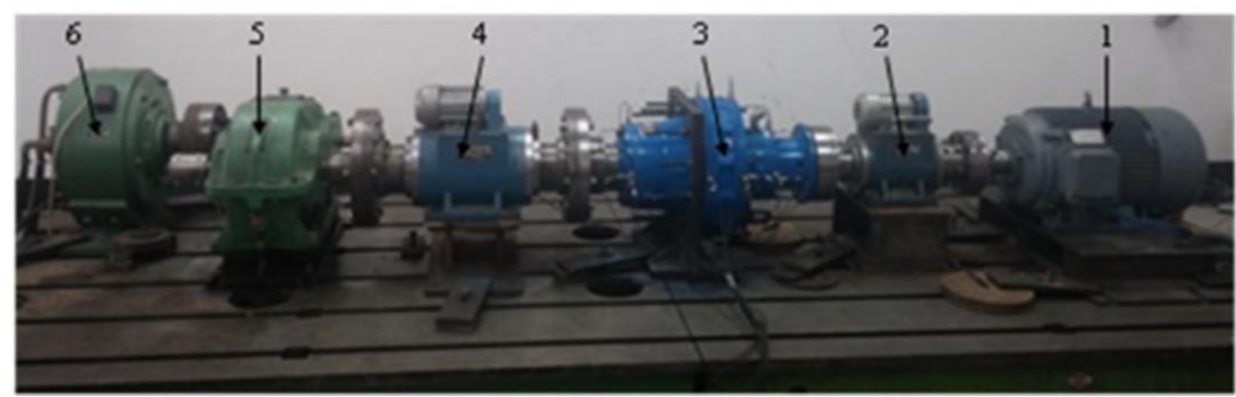

1. Three phase asynchronous motor; 2. Input torque speed sensor (I); 3. Prototype of the pin-cycloidal planetary reducer for the pitch; 4. Output torque speed sensor (II); 5. Speed increaser; 6. Magnetic particle brake. Fig 8:Experimental devices for pin-cycloidal planetary reducer for pitch of high-power wind turbines

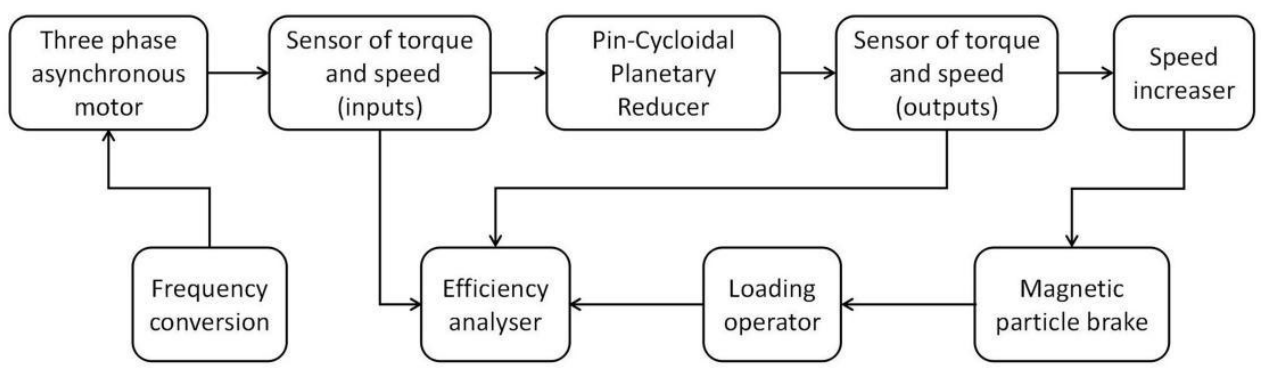

Fig 9:Test bench solution of the pin-cycloidal planetary reducer

The performance test of the reducer includes the following items and requirements:(1)No-load test. The forward and reverse no-load running-in tests were conducted at revolving speeds of $300 \mathrm{r} / \mathrm{min}$ and $475 \mathrm{r} / \mathrm{min}$, with each revolving speed operating for 30 minutes. The torque, revolving speed, transmission efficiency, vibration, noise, and casing temperature of the reducer were measured, and the reducer was checked for leakages and abnormal vibrations.(2)Loading test. A varying load of 9-45.45 N.m (there are a total of 5 load cases, i.e. 20\%, 40\%, 60\%, $80 \%$, and full load) was applied on the reducer at revolving speeds of $300 \mathrm{r} / \mathrm{min}$ and $475 \mathrm{r} / \mathrm{min}$, with each revolving speed operating for 30 minutes. The torque, revolving speed, transmission efficiency, vibration, noise, and casing temperature of the reducer were measured, and the reducer was checked for leakages or abnormal vibrations.(3)Overload operation. The load was increased step by step to $50 \mathrm{~N} \cdot \mathrm{m}$ and the reducer was kept running for 10 minutes to observe whether there was anything abnormal about the operation of the reducer.

Table 1 shows the performance test results of the prototype of the pin-cycloidal planetary reducer for the pitch of high-power wind turbines.

Table 1 Performance of the prototype of the pin-cycloidal planetary reducer for the pitch of high-power wind turbines

\begin{tabular}{|c|c|c|}
\hline Item & Stage-I & Stage-II \\
\hline$d_{p} / \mathrm{mm}$ & 330 & 176 \\
\hline Speed down ratio $i$ & 15 & 11 \\
\hline Motor speed $n / \mathrm{rpm}$ & \multicolumn{2}{|c|}{475} \\
\hline$P / \mathrm{kW}$ & \multicolumn{2}{|c|}{7500} \\
\hline$T / \mathrm{N} \cdot \mathrm{m}$ & \multicolumn{2}{|c|}{03} \\
\hline Transmission efficiency $\eta$ & \multicolumn{2}{|c|}{76} \\
\hline Noise $/ \mathrm{dB}$ & \multicolumn{2}{|c|}{} \\
\hline
\end{tabular}

ISSN: 0010-8189

www.converter-magazine.info 


\begin{tabular}{|c|c|} 
Temper $/^{\circ} \mathrm{C}$ & 46 \\
\hline
\end{tabular}

Figures 10 and 11 show the layout of vibration acceleration sensors of the reducer. A total of 14 measuring points were arranged on the prototype of the reducer to collect vibration signals in the corresponding axial and radial directions. Figure 12 shows the vibration mean square errors of the No. 12 measuring point in different working conditions.

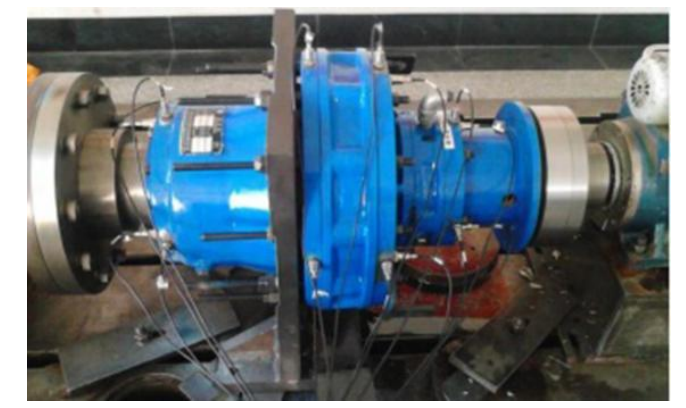

Fig 10:Layout of the vibration measuring points of the prototype

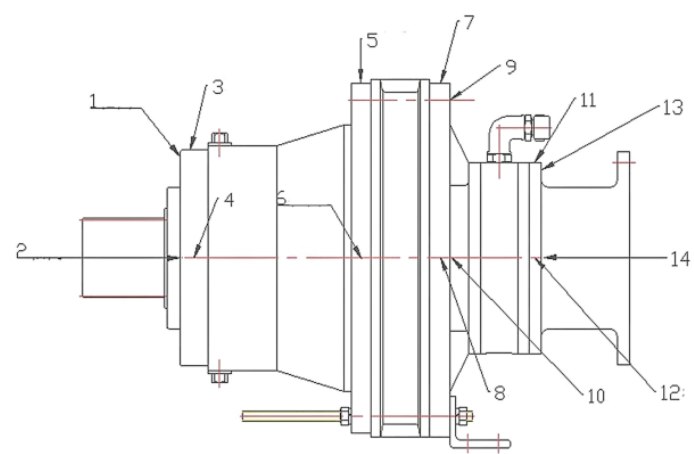

corresponding radial directionsmeasuring points:3,4,5,6,7,8,11,12.

corresponding axialmeasuring points: $1,2,9,10,13,14$

Fig 11:Layout of vibration acceleration sensors

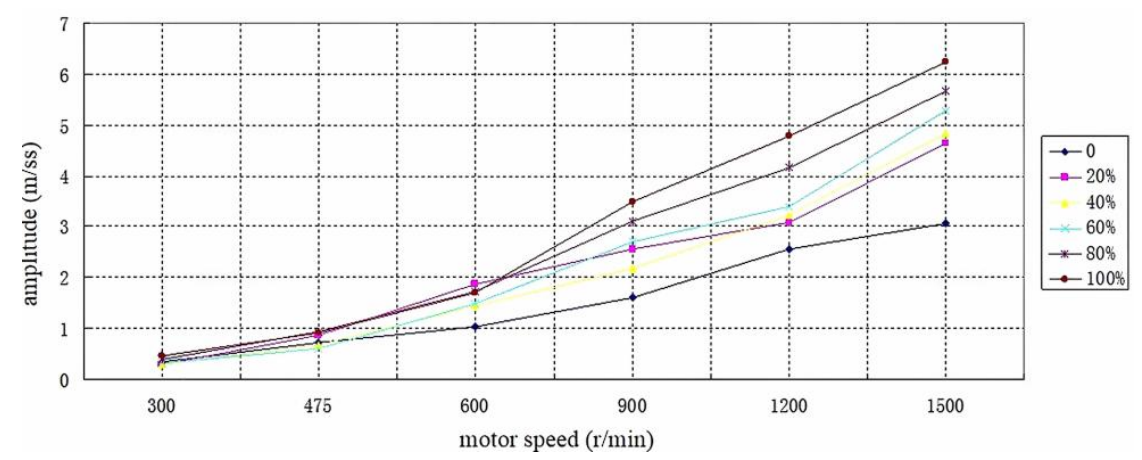

Fig 12:Vibration mean square errors of No. 12 measuring point in different working conditions

When the input speed is the rated speed of the prototype, i.e., $475 \mathrm{r} / \mathrm{min}$, the meshing frequency of the pin gear and the cycloidal gear in the stage-I deceleration system is $15.84 \mathrm{~Hz}$, and the meshing frequency of the pin gear and the cycloidal gear in the stage-II deceleration system is $1.5 \mathrm{~Hz}$, indicating that the prototype vibration source is mainly in stage I. According to the test data, in the six different load conditions, the prototype vibration is mainly reflected in the radial direction, and the vibration frequency is concentrated at two times $(32.5 \mathrm{~Hz})$ and twelve times $(190 \mathrm{~Hz})$ the meshing frequency. 
According to the frequency domain results obtained from the experiment and those from the dynamics simulation, the stage-I pin-cycloidal deceleration system is the main cause of vibration of the whole machine. Additionally, at a revolving speed of $475 \mathrm{r} / \mathrm{min}$ as well as in $20 \%, 40 \%, 80 \%$, and full load conditions, both the test results and simulation results reflect that the excitationfrequency is two times the meshing frequency of the stage-I cycloidal gear and pin gear $(32 \mathrm{~Hz})$.

To avoid resonance, the following measures can be taken:(1)Improve the contact rigidity of the pin gear and the cycloidal gear, reduce the elastic deformation of the cycloidal gear, and reduce the vibration caused by axial deformation.(2)Reduce the damping, ensure the bearing clearance, and reduce the energy consumed during the operation of the reducer.(3)Since low input revolving speed is likely to cause the reducer to resonate, in the actual operation, the low speed operation of the reducer should be avoided as much as possible and the revolving speed of the reducer should be increased to the rated speed as fast as possible.(4)The coupling between the elastic deformation of the cycloidal gear and the system is the main factor affecting the vibration of the reducer. The rigidity of the cycloidal gear can be appropriately increased in the design to decrease the vibration of the reducer.

\section{Conclusion}

In this study, pin-cycloidal planetary transmission with small tooth difference was applied to the electric pitch control system of wind turbines, a new two-stage pin-cycloidal planetary reducer with "two-teeth difference" was proposed, and the structural design and parameter optimization of this new reducer were carried out with the maximum carrying capacity of the whole machine as the objective function.

A mathematical model of the influences of the number of simultaneous meshing teeth and the load distribution between the teeth of pin-cycloidal transmission on the frictional work was established, and an equation more in accordance with the pin-cycloidal meshing efficiency in the actual meshing state was derived, which allowed the equation of the transmission efficiency of the whole machine to be derived.

Prototype testing was combined to verify the response of the excitation frequency to the amplitude-frequency characteristics of the whole system and to lay a foundation for further improving the dynamic performance of the whole machine.

\section{Acknowledgements}

This research was supported byEducational Commission of Liaoning Province of China (Grant No. JDL2017007).

\section{References}

[1] Jamal Muhammad Azfar, Shah Owaisur Rahman.Performance Evaluation of a $1 \mathrm{~kW}$ Variable PitchStraight Blade Vertical Axis Wind Turbine.Journal of Energy Resources TechnologyVolume 142, Issue 12, 2020.

[2] Wang Shaoping, Wang Bing, Shen Wangping.Research on Hळ Robust Control Strategy of Wind Turbine Hydraulic Variable Pitch System.IOP Conference Series: Earth and Environmental ScienceVolume 696, Issue 1. 2021.

[3] ISHIKAWA Shoichi.GDS-12 A Geometric Relationshipbetweenpitchcurvesandtoothprofilesinspurgearingwithvariableratio(gear design andsynthesis). The Proceedings of the Jsme International Conference on Motion and Power TransmissionsVolume II. 01. 202, 2001, pp: 484-487.

[4] He Weidong, Li Xin, Li Lixing. Study on New Pin-cycloidal Drive with High Load-capacity and High Transmission Efficiency. China Mechanical Engineering, 2005, 16(7): 565-569.

[5] He Weidong, Li Lixing, Li Jun. Force analysis on cycloidal gear of the RV drive used in robot. 1999, 2:49-53.

ISSN: 0010-8189 
[6] Zhang Zhan. Design and manufacture of gear boxes in the windy power industry. General Machinery, 2007: 31-34.

[7] Zhu Xiaolu. Handbook of Gear Design. Beijing: Chemical Industry Press, 2010, 554-555. Y. Su, J. Liu, Y.L. Huang, Research on entity recognition in online medical texts, Journal of Peking University (natural science edition), vol. 52, no. 1, pp: 1-9, 2016.

[8] FengChengzhou. Exploration on Pin-cycloidal Planetary Transmission Efficiency. Journal of Mechanical Transmission, 1979(2): 72. H.C. Lee, Y.Y. Hsu, H.Y. Kao, An Enhanced CRF-based System for Disease Name Entity Recognition and Normalization on BioCreative V DNER Task, In: Proceedings of the 5th BioCreative Challenge Evaluation Workshop, 2015.

[9] Liu Guangde, Xing Zuoxia, Li Ke et al. Research on Wind Turbine Electric Pitch System.ELECTRIC MACHINES \& CONTROL APPLICATION, 2006,33(10): 31-34.

[10] He Weidong, XunJianmin, BaoJunhua et al. Research on Calculation of Output Mechanism for Cycloid Reducer. Journal of DalianJiaotong University. 2010, 6:39-42. 